\title{
Improved Routing Protocol of Wireless Sensor Network Based on Wavelet Neural Network WANG Xiaopeng
}

\author{
Liaoning Geology Engineering Vocational College, Liaoning Dandong, 118000, China \\ wxp_1116@163.com
}

Keywords: wirless sensor network; LEACH; wavelet neural network; data fusion.

\begin{abstract}
The resource and especially the node energy of the wireless sensor network are limited. It can effectively prolong the lifetime of the wireless sensor network by reducing the energy consumption in the process of information collection and transmission as much as possible. Hereby a routing protocol improved algorithm model based on wavelet neural network was proposed for saving the energy and prolonging the network lifetime. The proposed algorithm model combined the hierarchical structure of the wavelet neural network with the clustering structure of the network routing protocol. In each cluster a wavelet neural network model is designed, which process a large number of raw data collected by the sensors and obtains a few data representing the data information features of raw data. Thus the transmission amount of data is reduced and the energy is saved. The simulation results show that the improved algorithm has a better performance in balancing node energy and prolonging the lifetime of the network compared with the low energy adaptive clustering hierarchy $(\mathrm{LEACH})$.
\end{abstract}

\section{Introduction}

Wireless sensor networks (WSN) is the new information monitoring system, which mainly by a large number of sensor nodes to complete data acquisition, transmission and processing [1]. The energy of sensor nodes mainly depend on the battery and the capacity of the battery is limited. Furthermore, the sensor nodes are often deployed in the relatively poor environment, which results in the battery of the sensor nodes is difficult to replace, the node energy is limited and cannot be added. For the WSN with limited energy, reducing energy consumption is an important measure to prolong the lifetime of the network. The energy of the wireless sensor network is mainly consumed in the wireless communication module. The energy of transmitting lbyte data information can be used to perform thousands of computer instructions [2], and the time consumed is less. Hereby, a routing protocol improved algorithm model based on wavelet neural network (WNN) was proposed to fusion the collected data by WNN in the routing protocol clustering structure. Thus there is only small amount of feature data transmit to the sink nodes, which can reduce the communication data volume of the nodes. This method can save the energy consumption of the sensor nodes and prolong the lifetime of the network effectively.

WSN and neural network (NN) has a lot of similar features, such as the sensor nodes has ability to handle data processing information, the WSN transmits the information among the sensors nodes by a certain rule. The features of the WSN just are same as the neuron and the synapses connect the neuron in NN. The application of the data fusion technology of WSN has the same function with the algorithm of NN, which deal with a large number of data by certain rules to obtain the results of the data feature. So the neural network algorithm can be applied to the WSN to achieve data fusion. Some related research results have been reported in recent years [3-5]. The proposed algorithm takes the data fusion technology to the routing layer of the WSN, Based on hierarchical routing, a new data fusion algorithm based on wavelet neural network (WNNDFA) was proposed. 


\section{Improvement of protocol algorithm based on wavelet neural network}

\section{The assumption conditions of the model}

There are many types of WSN in the application [6]. At first, we give the assumption conditions of the network model which was studied in this paper to illustrate the algorithm. The WSN model consists of N sensor nodes and the only one sink node. That is the WSN has only on base station and the WSN has the following properties: (1) The sensor nodes are randomly distributed in the monitoring area, once the sensor nodes is deployed, the sensor nodes will no longer be moved. All the sensor nodes are implemented to be a unique ID number and each sensor node in the network can verify their position coordinates information; (2) Each sensor node has the same type, the same initial energy and the same data processing capability, and the node energy is limited and cannot be added. Each sensor node of the cluster head consumes roughly the same energy; (3) The location of the sink node is fixed and deployed outside of the area of monitoring which has long distance to the monitoring area; (4) The energy of the sink node is infinite, and it can transmit the information directly to all nodes with enough power. The sink node is suitable for complex computational operation, and it need not worry about the energy consumption; (5) Each node in the wireless sensor network has the same structure as shown in Tab.1.

Table.1 Network node structure

\begin{tabular}{|c|c|c|c|}
\hline \multirow[b]{2}{*}{$\begin{array}{l}\text { Nodes have the remaining } \\
\text { energy }\end{array}$} & Communication unit & $\begin{array}{c}\text { Verification } \\
\text { unit }\end{array}$ & $\begin{array}{r}\text { Data } \\
\text { unit }\end{array}$ \\
\hline & $\begin{array}{l}\text { Simple } \\
\text { mechanism }\end{array}$ & $\begin{array}{l}\text { Position } \\
\text { information } \\
\text { RSSI information }\end{array}$ & $\begin{array}{l}\text { Data source } \\
\text { Adjacent node } \\
\text { number }\end{array}$ \\
\hline
\end{tabular}

\section{WNNDFA model}

LEACH clustering routing protocol [7] can improve the network performance compared with the flat routing protocol, so the proposed method based on LEACH clustering routing protocol. WNN is the combination of wavelet transform and NN [8] which can be divided into loose type and compact type. Compared with BP NN, WNN has faster convergence rate and higher convergence precision. Considering the WSN application, we choose compact WNN for data fusion. Related research has shown that, as long as the hidden layer node number is enough, the three layer WNN neural network has the ability to simulate any complex nonlinear mapping [9]. The WNN is applied to each cluster in the WSN, which construct the cluster WNN model. The collected data of each cluster is the input of the neuron function, after preprocessing, the data is transmitted to the cluster head node. The model of WNN is shown in Fig.1.

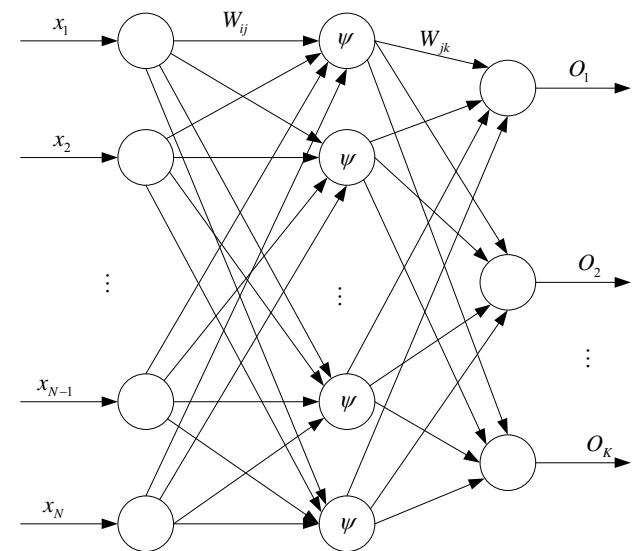

Fig.1 The model of WNN. 
Where $N$ is the total data type number that collected by each cluster node of the WSN, which is denoted by $x_{i}(i=1,2, \cdots, N)$, the corresponding input unit number of the WNN is $N$. The output unit number of WNN depends on the application of WSN, which can be designed according to the actual needs. The hidden layer unit number $J$ is designed according to the number of the training sample and the complexity of the sample. $W_{i j}(i=1,2, \cdots, N, j=1,2, \cdots, J)$ is the weights between the input layer and the hidden layer. $W_{j k}(j=1,2, \cdots, J, k=1,2, \cdots, K)$ is the weights between the hidden layer and the output layer. $\psi$ is the wavelet transform function in the hidden layer to improve the performance of $\mathrm{NN}$. The neuron number of hidden layer of the WNN has no theoretical basis. The method to determine the optimal number of the neuron of the hidden layer is trial method. Some empirical formula can be used to determine the neuron number of the hidden layer, such as the follows

$$
\begin{gathered}
J=\sqrt{N+K}+\alpha \\
J=\sqrt{N \times K}
\end{gathered}
$$

where $\alpha(0<\alpha<0)$ is a constant value. The wavelet function of WNN often choose the Morlet wavelet function which the wavelet transform can be given by

$$
\psi(x)=|a|^{1 / 2} \frac{x-b}{a} \exp \left(-\frac{(x-b)^{2}}{2 a^{2}}\right)
$$

where $a$ is the scale factor and $b$ is the shift factor of the wavelet transform.

As in Fig.1, in the cluster head node, the data would be processed by the hidden layer and the output layer of the WNN, and then the Eigen value can be obtained and further fusion by WNN to transmit to the sink node. The Data quantity of the Eigen value of the data far less than data itself, as a result, the transmit data quantity can be reduced and the energy consumption also can be reduced, the lifetime of the WSN can be extended.

\section{WNNDFA algorithm workflow}

In the proposed WNNDFA algorithm, some parameters of the WSN must be given in advance, such as the weights value of the WNN. Therefore, the WNN must be trained before working and after the clustering structure of the WSN begin stability. By training the WNN, the neural network can obtain a optimal weights and implement the steady state. For the energy of each node of the WSN is limited and cannot be added, WNNDFA completes the training operation in the internal of sink node of the WSN, which can save further save the energy consumption of the sensor nodes and prolong the lifetime of the network. The training flow chat of the WNN is shown in Fig.2.

The flow chat of the algorithm of WNNDFA is shown in Fig.3. In the wireless sensor network, the node's state and node position in the network should be determined firstly. When the WSN is initialized, the WSN begin to select the optimal cluster head and establish the clustering structure after the network node status is determined. At this time, the cluster head can obtain the related information of all the sensor nodes in the cluster. After the cluster structure is stable, the cluster head node sends the relevant information of all the nodes in the cluster to the sink node. The sink node constructs the structure of the WNN according to the network information and search the sample database which is matched with the information of the sensor nodes to train the WNN. After training, the WNN can obtain the related parameters. The sink node transmits the parameters such as the weights and the threshold of the WNN to the corresponding cluster nodes. Each cluster of the WSN can use the WNN model after training to implement the data fusion, and then it sends the data after processing to the sink node by shortest path. This method can reduce the transmission data quantity in the network to reduce the energy consumption of the sensor nodes, and the lifetime of the network can be prolonged. 


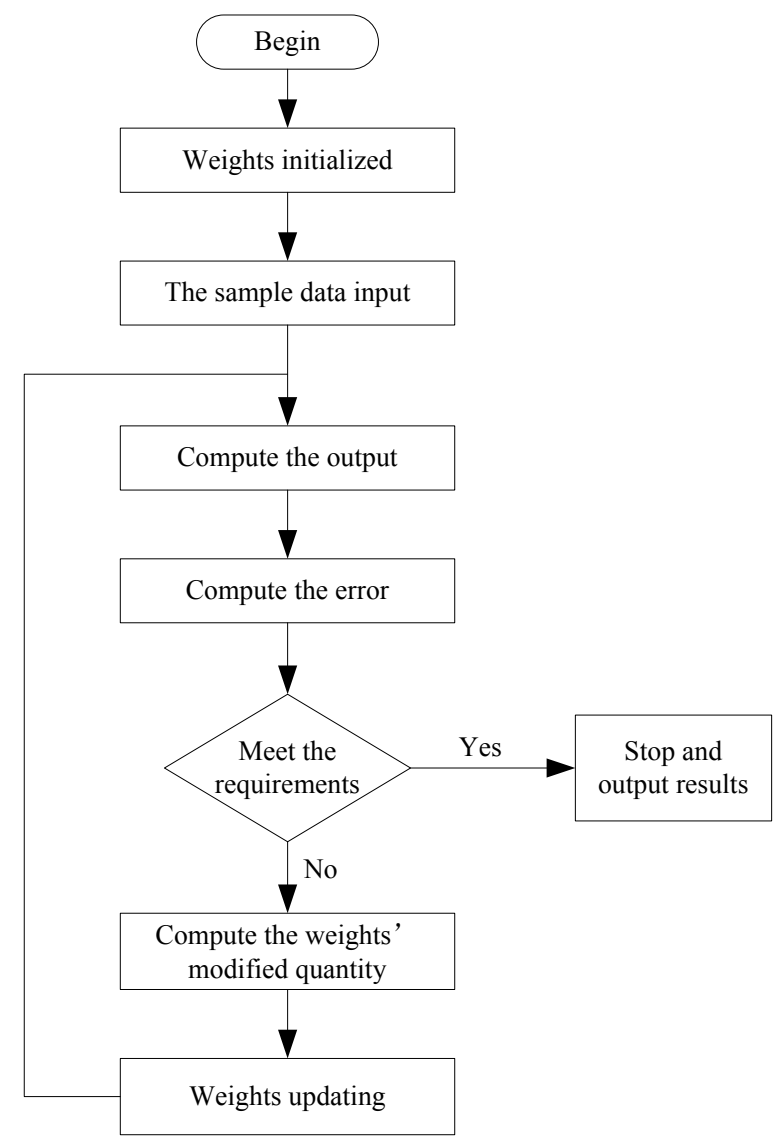

Fig. 2 The training flow of the WNN.

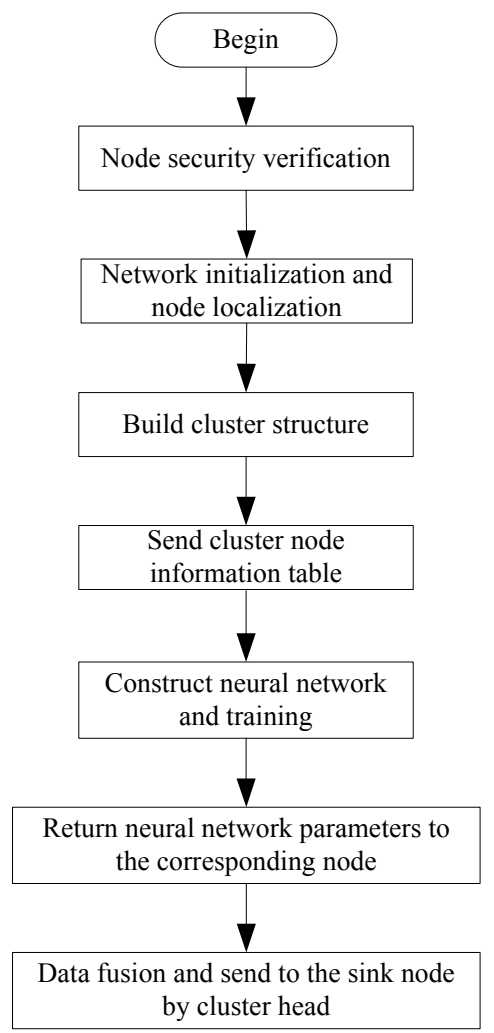

Fig.3 The workflow of the WNNDFA.

\section{Computer simulation}

The network simulation tool NS2 simulation software is adopted in the simulation to verify the algorithm of WNNDFA. The simulation environment settings are as follows: 150 identical nodes are randomly deployed in the area with range $120 \mathrm{~m} \times 120 \mathrm{~m}$. The sink node is arranged in a remote location to the sensing area with the initial energy $2 \mathrm{~J}$. The data package length is 350 byte and the wireless channel bandwidth is $1 \mathrm{Mbit} / \mathrm{s}$. The carrier frequency is $2.0 \mathrm{GHz}$. Energy consumed by sending and receiving data is $45 \mathrm{~nJ} / \mathrm{bit}$. The parameters of the $\mathrm{WNN}$ are initialized random and the maximum iterative times is 1200 .

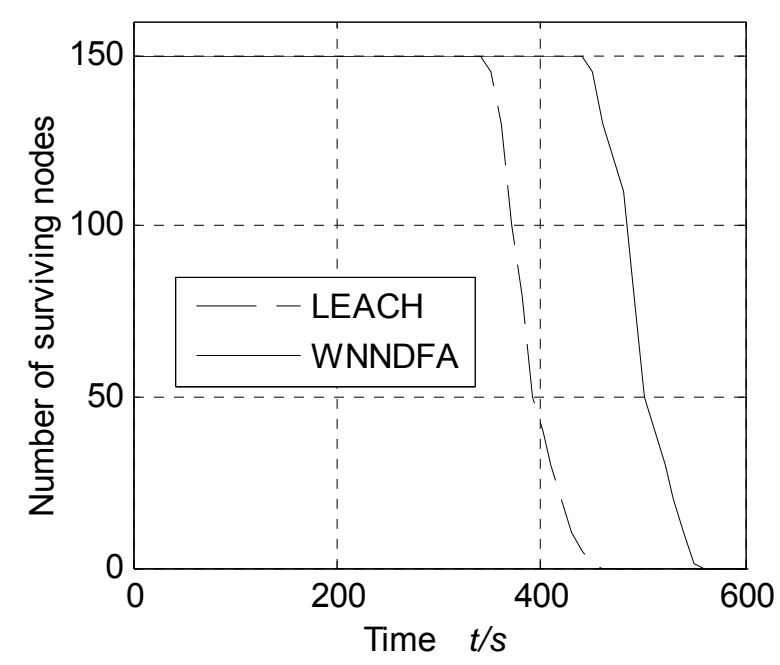

Fig.4 Network surviving node number

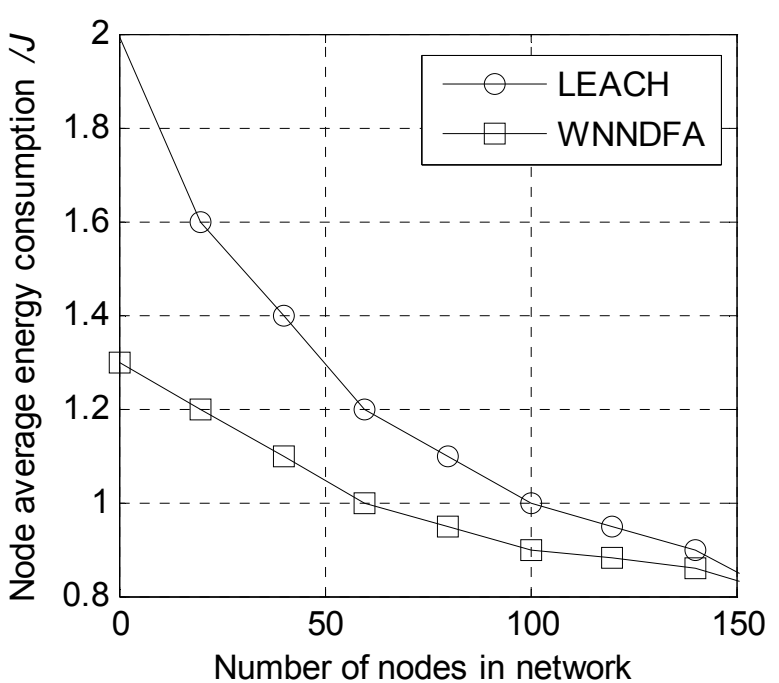

Fig.5 Node average energy consumption 
Fig. 4 shows the comparison of the survival number of nodes in the network with LEACH algorithm and WNNDFA algorithm. Fig. 5 shows the average energy consumption of nodes in the network with LEACH algorithm and WNNDFA algorithm when the nodes number different. The results show that LEACH algorithm begins to appear node death at 350s and WNNDFA algorithm begins to appear node death at 450s. The node death is gradually accelerated and there are no survival nodes at 460s for LEACH algorithm. Compared with LEACH algorithm, the node death rate is slower in WNNDFA algorithm. Furthermore, the average energy consumption of the WNNDFA algorithm is significantly lower than that of the LEACH algorithm. The results prove that WNNDFA algorithm improve the performance of the WSN effectively

\section{Conclusion}

For wireless sensor networks, energy limited is an important indicator. How to reduce energy consumption and prolong the network life cycle is an important research content. This work proposed an improved method which the WNN is applied to the clustering structure of WSN and the protocol improvement WNNDFA algorithm is obtained. By fusion processing of the collected data in the network, the transmission data quantity is reduced effectively, and then the energy consumption of the total network is reduced, as a result, the purpose of prolonging the network lifetime is achieved.

\section{References}

[1] L.M. SUN, J.Z. LI, Y. CHEN. Wireless sensor network [M]. Beijing: Tsinghua University press, 2005.

[2] R. BI, J.Z. LI. Energy efficient top-k monitoring algorithm in wireless sensor network [J]. Journal of Computer Research and Development, 2014, 51(11): 2361-2373.

[3] M. BOCCA, J. TOIVOLA, L. M. ERIKSSON, et al. Structure health monitoring in wireless sensor networks by the embedded goertzel algorithm [C] // Proc of the $2^{\text {nd }}$ Int Conf on Cyber-Physical Systems (ICCPS). Alamitos, CA: IEEE Computer Society, 2011:206-214.

[4] S. MICHEL, L. GRAVANO, A. MARIAN. Optimizing top-k selection queries over multimedia repositories [J]. IEEE Trans on Knowledge and Data Engineering, 2004, 16(8): 992-1009.

[5] M. J. WU, J. L. XU, X. Y. TANG, et al. Top-k monitoring in wireless sensor network [J]. IEEE Trans on Knowledge and Data Engineering, 2007, 19(7): 962-976.

[6] K. J. MAO, C. L. FAN, F. YE, et al. Node localization algorithm in wireless sensor network based on SVM [J]. Journal of Computer Research and Development, 2014, 51(11): 2427-2436.

[7] J. Z. LI. Foreword special issue in wireless sensor networks [J]. Journal of Software, 2007, 18(8): 1077-1079.

[8] N. LU, Z. H. XIAO, X. Q. FU. Vibration fault diagnosis of hydroelectric generating unit based on ACO-initialized wavelet network [J]. Journal of Hydroelectric Engineering, 2014, 33(2): 251-258.

[9] C.H. JU, X. N. GUO. Data stream coincidence characteristic clustering method based on wavelet network [J]. Computer Engineering and Design, 2012, 33(3): 1145-1149. 